\title{
A CLASS OF LOCALIZED SOLUTIONS OF THE LINEAR AND NONLINEAR WAVE EQUATIONS
}

\author{
LUBOMIR M. KOVACHEV AND DANIELA A. GEORGIEVA
}

\section{Communicated by Boris Konopeltchenko}

\begin{abstract}
Following the tradition of the nano and picosecond optics, the basic theoretical studies continue to investigate the processes of propagation of femtosecond and attosecond laser pulses through the corresponding envelope equation for narrow-band laser pulses, working in paraxial approximation. We should point out here that this approximation is not valid for large band pulses. In air due to the small dispersion the wave equation as well as the $3 D+1$ amplitude equation describe more accurate the pulse dynamics. New exact localized solutions of the linear wave and amplitude equations are presented. The solutions discover non-paraxial semi-spherical diffraction of single-cycle and half-cycle laser pulses and a new class of spherically symmetric solutions of the wave equation. The propagation of large band optical pulses in nonlinear vacuum is investigated also in the frame of a system of nonlinear wave vector equations. We obtained exact vector solution with its own angular momentum in the form of a shock wave.
\end{abstract}

\section{Introduction}

With the progress of laser innovations it is very important to study the localized waves, especially pulses which admit only few cycles under the envelope and pulses in half-cycle regime. One important experimental result is that even in femtosecond region, the waist (transverse size) of an initially non modulated laser pulse continues to satisfy the Fresnel's law of diffraction. The parabolic diffraction equation governing Fresnel's evolution of a monochromatic wave in continuous regime (CW regime) is suggested for first time by Leontovich and Fock [5, 12, 13]

$$
\frac{\partial^{2} w}{\partial x^{2}}+\frac{\partial^{2} w}{\partial y^{2}}+4 \mathrm{i} k_{0} \frac{\partial w}{\partial z}=0
$$

The solutions of equation (1) possess circular fundamental Gaussian mode [3] as well as higher-order modes, such as Laplace-Gauss $[1,9,17]$, Helmholtz-Gauss and Bessel-Gauss [2, 6, 7,9] beams. On the other hand the optics of laser pulses, especially in the femtosecond (fs) region operates with strongly polychromatic waves 
and their spectral width $\Delta k_{z}$ can reach values of order of the main wave number $k_{0}$. There are additional possibilities if we consider a fs pulse to admit approximately equal size in $x, y$ and $z$ directions (Light Bullet or LB), or relatively large transverse and small longitudinal size (Light Disk or LD). The evolution of so generated LB and LD in linear or nonlinear regime is quite different from the propagation of light beams and this has drawn the researchers' attention with an unexpected dynamical behavior. In [11] we have found new exact solution of the wave equation considering initial form of Gaussian light bullet having semi-spherical, non paraxial diffraction in air in the case when the pulse admits few cycles only under the envelope. We suggest a simple Courant - Hilbert ansatz in form

$$
E(x, y, z, t)=V(x, y, z, t) \exp \left(\mathrm{i} k_{0}(z-v t)\right)
$$

applied to the wave equation

$$
\Delta E-\frac{1}{v^{2}} \frac{\partial^{2} E}{\partial t^{2}}=0
$$

The corresponding diffraction equation of the amplitude function $V$ is than

$$
-2 \mathrm{i} k_{0}\left(\frac{\partial V}{\partial z}+\frac{1}{v} \frac{\partial V}{\partial t}\right)=\Delta V-\frac{1}{v^{2}} \frac{\partial^{2} V}{\partial t^{2}}
$$

where $v$ is the phase velocity (or the group velocity in air when the second order of dispersion is neglected). The latest developments in spectroscopy of dielectrics and semiconductors allow optical pulses with non oscillating nature, so called HalfCycle Pulses (HCP's) [8, 18]. In terms of our ansatz (2) this implies, that for the HCP's the longitudinal spatial $\Delta z$ or temporal $\Delta t$ shape of the amplitude envelope $V$ should be shorter than the main wavelength $\lambda_{0}=2 \pi / k_{0}$ or time period $T_{0}=$ $2 \pi / \omega_{0}$.

In this paper we have obtained a class of spherically symmetric analytical solutions of the wave (3) and amplitude (4) equations. The main result is that the solutions strongly depend on their initial localization. When the initial pulse is localized near one plane wave two kind of unidirectional wave propagation are found: paraxial and non-paraxial. When the initial localized wave is spherically symmetric the diffraction is radial with formation of inside and outside fronts. In all cases the propagation of light pulses is not stable - enlargement and decrease of the amplitude is observed. In order to find stable pulse propagation we investigate mainly large band pulses propagating in two types homogenous media - air and nonlinear vacuum. In air a scalar stable Lorentz type soliton was obtained [10] while in nonlinear vacuum we have obtained a vector solution with its own angular momentum. 


\section{Linear Regime of Optical Pulses}

The experiments with ultrashort fs and attosecond pulses as well as the numerical calculations [16] point out the main problem in the diffraction theory: namely, whether it is possible to build such a $3 D+1$ diffraction (and dispersion) model that corresponds to the following experimental results: $a$ ) at one diffraction length the spot of any spectrally limited laser pulse satisfies the Fresnel's diffraction and $b$ ) at several diffraction lengths one or two cycle optical pulses diffract semi-spherically. Approximating up to second order of dispersion the linear Diffraction - Dispersion Equation (DDE) governing the propagation of the pulse is [11]

$$
-2 \mathrm{i} k_{0}\left(\frac{\partial A}{\partial z}+\frac{1}{v} \frac{\partial A}{\partial t}\right)=\Delta A-\frac{1+\beta}{v^{2}} \frac{\partial^{2} A}{\partial t^{2}}
$$

where $\beta=k^{\prime \prime} k_{0} v^{2}$ is a number giving the influence of the second order of dispersion. In dispersionless media is also obtained the following Diffraction Equation

$$
-2 \mathrm{i} k_{0}\left(\frac{\partial V}{\partial z}+\frac{1}{v} \frac{\partial V}{\partial t}\right)=\Delta V-\frac{1}{v^{2}} \frac{\partial^{2} V}{\partial t^{2}} .
$$

In air $\beta \simeq 2.1 \times 10^{-5} \simeq 0$, and hence $\operatorname{DDE}(5)$ is equal to the differential equation (6), and till hundred diffraction lengths exist only diffraction problems. This means that we can use the approximation $\beta \simeq 0$ and we can investigate the differential equation (6) within these distances. As it was mentioned in the Introduction the differential equation (6) can be obtained directly from the wave equation using the ansatz (2). Thus, solving the amplitude equation (6) and multiplying with the main phase, we can obtain exact solution of the wave equation.

DDE (5) and DE (6) are solved by applying the spatial Fourier transform to the components of the amplitude functions $A$ and $V$. The fundamental solutions of the Fourier images $\hat{A}$ and $\hat{V}$ in $\left(k_{x}, k_{y}, k_{z}, t\right)$ space are correspondingly

$$
\begin{aligned}
& \hat{A}\left(k_{x}, k_{y}, k_{z}, t\right)=\hat{A}\left(k_{x}, k_{y}, k_{z}, 0\right) \\
& \times \exp \left(\frac{\mathrm{i} v}{\beta+1}\left(k_{0} \pm \sqrt{k_{0}^{2}+(\beta+1)\left(k_{x}^{2}+k_{y}^{2}+k_{z}^{2}-2 k_{0} k_{z}\right)}\right) t\right) \\
& \begin{array}{r}
\hat{V}\left(k_{x}, k_{y}, k_{z}, t\right)=\hat{V}\left(k_{x}, k_{y}, k_{z}, 0\right) \\
\times \exp \left(\mathrm{i} v\left(k_{0} \pm \sqrt{k_{x}^{2}+k_{y}^{2}+\left(k_{z}-k_{0}\right)^{2}}\right) t\right) .
\end{array}
\end{aligned}
$$


In the case when $\beta \simeq 0$ the fundamental solution (7) is equal to (8) and therefore we shall investigate only (8). The exact solution of equation (6) can be obtained by applying the backward Fourier transform

$$
V=F^{-1}\left[\hat{V}\left(k_{x}, k_{y}, k_{z}, 0\right) \exp \left(\mathrm{i} v\left(k_{0} \pm \sqrt{k_{x}^{2}+k_{y}^{2}+\left(k_{z}-k_{0}\right)^{2}}\right) t\right)\right]
$$

or in detail

$$
\begin{aligned}
V=\frac{1}{(2 \pi)^{3}} \int_{-\infty}^{\infty} \int_{-\infty}^{\infty} \int_{-\infty}^{\infty} \hat{V}\left(k_{x}, k_{y}, k_{z}, 0\right) \\
\quad \times \exp \left(\mathrm{i} v\left(k_{0} \pm \sqrt{k_{x}^{2}+k_{y}^{2}+\left(k_{z}-k_{0}\right)^{2}}\right) t\right) \\
\quad \times \exp \left(-\mathrm{i}\left(x k_{x}+y k_{y}+z k_{z}\right)\right) \mathrm{d} k_{x} \mathrm{~d} k_{y} \mathrm{~d} k_{z} .
\end{aligned}
$$

Substituting $k_{z}-k_{0}=\hat{k}_{z}$ in (10) the latter takes the form

$$
\begin{aligned}
V= & \frac{1}{(2 \pi)^{3}} \exp \left(\mathrm{i} k_{0} v t\right) \int_{-\infty}^{\infty} \int_{-\infty}^{\infty} \int_{-\infty}^{\infty} \hat{V}\left(k_{x}, k_{y}, \hat{k}_{z}+k_{0}, 0\right) \\
& \times \exp \left( \pm \mathrm{i} v t \sqrt{k_{x}^{2}+k_{y}^{2}+\hat{k}_{z}^{2}}\right) \exp \left(-\mathrm{i}\left(x k_{x}+y k_{y}+z \hat{k}_{z}\right)\right) \mathrm{d} k_{x} \mathrm{~d} k_{y} \mathrm{~d} \hat{k}_{z} .
\end{aligned}
$$

We can solve the backward Fourier transform in (11), if there is the possibility the initial Fourier image $\hat{V}\left(k_{x}, k_{y}, \hat{k}_{z}+k_{0}, 0\right)$ to be presented as a function of kind $f\left(k_{0}\right) \hat{V}^{*}\left(k_{x}, k_{y}, \hat{k}_{z}, 0\right)$. Thus (11) becomes

$$
\begin{aligned}
V= & \frac{1}{(2 \pi)^{3}} \exp \left(\mathrm{i} k_{0} v t\right) f\left(k_{0}\right) \int_{-\infty}^{\infty} \int_{-\infty}^{\infty} \int_{-\infty}^{\infty} \hat{V}^{*}\left(k_{x}, k_{y}, \hat{k}_{z}, 0\right) \\
& \times \exp \left( \pm \mathrm{i} v t \sqrt{k_{x}^{2}+k_{y}^{2}+\hat{k}_{z}^{2}}\right) \exp \left(-\mathrm{i}\left(x k_{x}+y k_{y}+z \hat{k}_{z}\right)\right) \mathrm{d} k_{x} \mathrm{~d} k_{y} \mathrm{~d} \hat{k}_{z}
\end{aligned}
$$

where the whole integrand in (12) depends on the same translated wave number $\hat{k}_{z}$ and can be solved analytically.

\subsection{Gaussian Light Bullet}

We can begin with a solution obtained for first time in [11] in order to illustrate in detail the method of finding localized solution of the differential equation (6) and the wave equation (3) possessing a finite energy. The convolution problem (8) was solved for initial Gaussian light bullet of the kind $V(x, y, z, 0)=$ 
$\exp \left(-\left(x^{2}+y^{2}+z^{2}\right) / 2 r_{0}^{2}\right)$. In this case the $3 D$ backward Fourier transform (11) becomes

$$
\begin{aligned}
V= & \frac{1}{(2 \pi)^{3}} \exp \left(-\frac{k_{0}^{2} r_{0}^{2}}{2}-\mathrm{i} k_{0}(z-v t)\right) \\
& \times \int_{-\infty}^{\infty} \int_{-\infty}^{\infty} \int_{-\infty}^{\infty} \exp \left(-\frac{\left(k_{x}^{2}+k_{y}^{2}+\hat{k}_{z}^{2}\right) r_{0}^{2}}{2}\right) \times \exp \left( \pm \mathrm{i} v t \sqrt{k_{x}^{2}+k_{y}^{2}+\hat{k}_{z}^{2}}\right) \\
& \times \exp \left(-\mathrm{i}\left(x k_{x}+y k_{y}+\left(z-\mathrm{i} r_{0}^{2} k_{0}\right) \hat{k}_{z}\right)\right) \mathrm{d} k_{x} \mathrm{~d} k_{y} \mathrm{~d} \hat{k}_{z}
\end{aligned}
$$

which in spherical coordinates can be presented in the following way

$$
\begin{aligned}
V=\frac{1}{2 \pi^{2} \hat{r}} \exp ( & \left.-\frac{k_{0}^{2} r_{0}^{2}}{2}-\mathrm{i} k_{0}(z-v t)\right) \\
& \times \int_{0}^{\infty} \hat{k}_{r} \exp \left(-\frac{\hat{k_{r}^{2} r_{0}^{2}}}{2}\right) \exp \left( \pm \mathrm{i} v t \hat{k}_{r}\right) \sin \left(\hat{r} \hat{k}_{r}\right) \mathrm{d} \hat{k}_{r}
\end{aligned}
$$

where $\hat{k}_{r}=\sqrt{k_{x}^{2}+k_{y}^{2}+\hat{k}_{z}^{2}}$ and $\hat{r}=\sqrt{x^{2}+y^{2}+\left(z-\mathrm{i} r_{0}^{2} k_{0}\right)^{2}}$.

The corresponding amplitude solution is

$$
\begin{aligned}
V(x, y, z, t)= & \frac{\mathrm{i}}{2 \hat{r}} \exp \left(-\frac{k_{0}^{2} r_{0}^{2}}{2}-\mathrm{i} k_{0}(z-v t)\right) \\
& \times\left(\mathrm{i}(v t+\hat{r}) \exp \left(-\frac{1}{2 r_{0}^{2}}(v t+\hat{r})^{2}\right) \operatorname{erfc}\left(\frac{\mathrm{i}}{\sqrt{2} r_{0}}(v t+\hat{r})\right)\right. \\
& \left.-\mathrm{i}(v t-\hat{r}) \exp \left(-\frac{1}{2 r_{0}^{2}}(v t-\hat{r})^{2}\right) \operatorname{erfc}\left(\frac{\mathrm{i}}{\sqrt{2} r_{0}}(v t-\hat{r})\right)\right) .
\end{aligned}
$$

Multiplying (15) with the main phase we find actually an exact solution of the wave equation (3)

$$
\begin{aligned}
E(x, y, z, t)= & \frac{\mathrm{i}}{2 \hat{r}} \exp \left(-\frac{k_{0}^{2} r_{0}^{2}}{2}\right) \\
& \times\left(\mathrm{i}(v t+\hat{r}) \exp \left(-\frac{1}{2 r_{0}^{2}}(v t+\hat{r})^{2}\right) \operatorname{erfc}\left(\frac{\mathrm{i}}{\sqrt{2} r_{0}}(v t+\hat{r})\right)\right. \\
& \left.-\mathrm{i}(v t-\hat{r}) \exp \left(-\frac{1}{2 r_{0}^{2}}(v t-\hat{r})^{2}\right) \operatorname{erfc}\left(\frac{\mathrm{i}}{\sqrt{2} r_{0}}(v t-\hat{r})\right)\right)
\end{aligned}
$$

Substituting the time variable $t=0$ in equation (16) the initial solution of the wave equation is $E(x, y, z, 0)=\exp \left(\mathrm{i} k_{0} z\right) \exp \left(-\left(x^{2}+y^{2}+z^{2}\right) / 2 r_{0}^{2}\right)$. All analytical 
and numerical solutions of the wave equation (3) obtained from the initial conditions of kind $E(x, y, z, 0)=\exp \left(\mathrm{i} k_{0} z\right) V(x, y, z, 0)$, where $V(x, y, z, 0)$ is a three dimensional localized smooth function, produce an unidirectional propagation in $z$ direction. The wave equation (3) is of hyperbolic type, while the amplitude equation (6) is of parabolic type and a initial value problem (11) can be solved. One method for finding spherically symmetric solutions of the wave equation appears hire. Now, let the initial amplitude function $V$ of the amplitude equation is a product of a three dimensional localized function, multiplied by a plane wave with opposite direction

$$
V(x, y, z, 0)=\exp \left(-\mathrm{i} k_{0} z\right) V^{*}(x, y, z, 0)
$$

where $V^{*}$ is a spherically symmetric function. Then the corresponding initial amplitude function of the wave equation becomes $E(x, y, z, 0)=V^{*}(x, y, z, 0)$ and by solving initial value problem (6) with initial conditions of kind (17), we can find exact spherically symmetric solutions of the wave equation (3).

\subsection{Spherically Symmetric Finite Energy Solutions of the Wave Equation}

One watchful analysis of the solution of the amplitude function $V$ in equation (11) reveals that all functions under the integral depend on the translated wave number $\hat{k}_{z}$, except the Fourier image of the initial function $\hat{V}\left(k_{x}, k_{y}, \hat{k}_{z}+k_{0}, 0\right)$. We can use the Transition theorem from the Fourier optics to present the above mentioned function $\hat{V}\left(k_{x}, k_{y}, \hat{k}_{z}+k_{0}, 0\right)$ as a function only of the translated wave number $\hat{k}_{z}: f\left(k_{0}\right) \hat{V}^{*}\left(k_{x}, k_{y}, \hat{k}_{z}, 0\right)$. Formally, this corresponds to the use of the initial conditions in the form

$$
V(x, y, z, 0)=V^{*}(x, y, z, 0) \exp \left(-\mathrm{i} k_{0} z\right) .
$$

Applying the Transition theorem to (18), we obtain the following expression of the Fourier image

$$
F[V(x, y, z, 0)]=\hat{V}\left(k_{x}, k_{y}, k_{z}-k_{0}, 0\right)=\hat{V}^{*}\left(k_{x}, k_{y}, \hat{k}_{z}, 0\right) .
$$

Thus, all functions in the backward Fourier transform (11) depend only on translated wave number $\hat{k}_{z}$

$$
\begin{aligned}
V= & \frac{1}{(2 \pi)^{3}} \exp \left(\mathrm{i} k_{0}(v t-z)\right) \int_{-\infty}^{\infty} \int_{-\infty}^{\infty} \int_{-\infty}^{\infty} \hat{V}^{*}\left(k_{x}, k_{y}, \hat{k}_{z}, 0\right) \\
& \times \exp \left( \pm \mathrm{i} v t \sqrt{k_{x}^{2}+k_{y}^{2}+\hat{k}_{z}^{2}}\right) \exp \left(-\mathrm{i}\left(x k_{x}+y k_{y}+z \hat{k}_{z}\right)\right) \mathrm{d} k_{x} \mathrm{~d} k_{y} \mathrm{~d} \hat{k}_{z} .
\end{aligned}
$$


In this paper we shall consider spherically-symmetric functions of kind

$$
V^{*}(x, y, z, 0)=V^{*}(r, 0)
$$

where $r=\sqrt{x^{2}+y^{2}+z^{2}}$.

This means that the backward radial Fourier transform can be written as

$$
\begin{aligned}
V=\frac{1}{2 \pi^{2} r} \exp \left(\mathrm{i} k_{0}(v t\right. & -z)) \\
& \times \int_{0}^{\infty} \hat{k}_{r} \hat{V}^{*}\left(\hat{k}_{r}, 0\right) \exp \left( \pm \mathrm{i} v t \hat{k}_{r}\right) \sin \left(r \hat{k}_{r}\right) \mathrm{d} \hat{k}_{r} .
\end{aligned}
$$

So, now the method of finding finite energy spherically symmetric solutions of the wave equation becomes obvious. Using an initial condition in the form $V=$ $V^{*} \exp \left(-\mathrm{i} k_{0} z\right)$, where $V^{*}$ is a localized function and $V^{*}(r) \mapsto 0$ where $r \mapsto \infty$, as a first step we find its Fourier image $\hat{V}^{*}\left(\hat{k}_{r}, 0\right)$. Then solving the backward radial Fourier transform (21) we can find the solution of the corresponding the differential equation (6). Multiplying the solution of the differential equation (6) with the main phase (Courant-Hilbert ansatz (2)) we practically obtain an exact solution of the wave equation (3).

\subsubsection{Localized Algebraic Function of the Kind $V^{*}=1 /\left(1+r^{2} / r_{0}^{2}\right)$}

We demonstrate here how through this method we can obtain a finite energy solution for initial algebraic localized function of the kind

$$
V^{*}(x, y, z, t=0)=1 /\left(1+r^{2} / r_{0}^{2}\right) .
$$

The 3D Fourier expression of (22) in spherical variables is

$$
\hat{V}^{*}\left(k_{r}, t=0\right)=\frac{\pi}{2 k_{r}} \exp \left(-r_{0} k_{r}\right) .
$$

Hence, after solving the spectral kernels (21) of (6) the corresponding solution of the amplitude equation is

$$
V(x, y, z, t)=\exp \left(-\mathrm{i} k_{0}(z-v t)\right)\left(\frac{r^{2}}{r_{0}^{2}}+\left(1+\frac{\mathrm{i} v t}{r_{0}}\right)^{2}\right)^{-1}
$$

Then again by multiplying with the main phase, the corresponding finite energy solution of the wave equation (3) becomes

$$
E(x, y, z, t)=\left(\frac{r^{2}}{r_{0}^{2}}+\left(1+\frac{\mathrm{i} v t}{r_{0}}\right)^{2}\right)^{-1} .
$$


2.2.2. Localized Algebraic Function of the Kind $V^{*}=1 /\left(\left(1+r^{2} / r_{0}^{2}\right)^{2}\right)$

The function

$$
V^{*}(r)=1 /\left(1+r^{2} / r_{0}^{2}\right)^{2}
$$

has a Fourier image

$$
\hat{V}^{*}\left(k_{r}\right)=\frac{1}{4} \pi r_{0} \exp \left(-r_{0} k_{r}\right) .
$$

Hence, the corresponding solution of the amplitude equation is

$$
V(x, y, z, t)=\exp \left(-\mathrm{i} k_{0}(z-v t)\right)\left(\frac{2\left(r_{0}+\mathrm{i} t v\right)}{\left(r^{2}+\left(r_{0}+\mathrm{i} t v\right)^{2}\right)^{2}}\right)
$$

and the corresponding wave solution is

$$
E(x, y, z, t)=\frac{2\left(r_{0}+\mathrm{i} t v\right)}{\left(r^{2}+\left(r_{0}+\mathrm{i} t v\right)^{2}\right)^{2}} .
$$

\subsubsection{Localized Algebraic Function of the Kind $V^{*}=1 /\left(\left(1+r^{2}\right)^{4}\right)$}

The Fourier image of the function

$$
V^{*}(r)=1 /\left(\left(1+r^{2}\right)^{4}\right)
$$

is the following expression

$$
\hat{V}^{*}=\frac{1}{96} \pi\left(3+k_{r}^{2}+3 k_{r}\right) \exp \left(-k_{r}\right) .
$$

The solution $V$ of the differential equation (6) becomes

$$
\begin{array}{r}
V(x, y, z, t)=6 \exp \left(-\mathrm{i} k_{0}(z-v t)\right) \\
\times\left(\frac{(8+29 \mathrm{i} t v)+t v\left(-r^{2}+t^{2} v^{2}\right)\left(-\mathrm{i} r^{2}+t v(8+\mathrm{i} t v)\right)}{\left(r^{2}+(1+\mathrm{i} t v)^{2}\right)^{4}}\right. \\
\left.-\frac{2 t v\left(-3 \mathrm{i} r^{2}+t v(20+13 \mathrm{i} t v)\right)}{\left(r^{2}+(1+\mathrm{i} t v)^{2}\right)^{4}}\right)
\end{array}
$$

and the corresponding wave solution is

$$
\begin{aligned}
E(x, y, z, t)= & 6\left(\frac{(8+29 \mathrm{i} t v)+t v\left(-r^{2}+t^{2} v^{2}\right)\left(-\mathrm{i} r^{2}+t v(8+\mathrm{i} t v)\right)}{\left(r^{2}+(1+\mathrm{i} t v)^{2}\right)^{4}}\right. \\
& \left.-\frac{2 t v\left(-3 \mathrm{i} r^{2}+t v(20+13 \mathrm{i} t v)\right)}{\left(r^{2}+(1+\mathrm{i} t v)^{2}\right)^{4}}\right) .
\end{aligned}
$$


2.2.4. Localized Algebraic Function of the Kind $V^{*}=24\left(1-r^{2}\right) /\left(1+r^{2}\right)^{4}$

The function

$$
V^{*}(r)=24\left(1-r^{2}\right) /\left(1+r^{2}\right)^{4}
$$

has a Fourier image

$$
\frac{1}{2} \pi k_{r}^{2} \exp \left(-k_{r}\right)
$$

Hence, the solution $V$ of the differential equation (6) is

$$
\begin{aligned}
V(x, y, z, t)= & \frac{3 \mathrm{i}}{4 \pi r} \exp \left(-\mathrm{i} k_{0}(z-v t)\right) \\
& \times\left(\frac{1}{(v t+r-\mathrm{i})^{4}}-\frac{1}{(-v t+r+\mathrm{i})^{4}}\right)
\end{aligned}
$$

and the corresponding wave solution is

$$
E(x, y, z, t)=\frac{3 \mathrm{i}}{4 \pi r}\left(\frac{1}{(v t+r-\mathrm{i})^{4}}-\frac{1}{(-v t+r+\mathrm{i})^{4}}\right) .
$$

\subsubsection{Localized Algebraic Function of the Kind $V^{*}=2 r\left(3-r^{2}\right) /\left(1+r^{2}\right)^{3}$}

The function

$$
V^{*}(r)=2 r\left(3-r^{2}\right) /\left(1+r^{2}\right)^{3}
$$

has a Fourier image

$$
\frac{1}{2} \pi k_{r} \exp \left(-k_{r}\right)
$$

Hence, the solution $V$ of the differential equation (6) is

$$
V(x, y, z, t)=\frac{1}{r} \exp \left(-\mathrm{i} k_{0}(v t-z)\right)\left(\frac{1}{(v t-r-\mathrm{i})^{3}}-\frac{1}{(v t+r-\mathrm{i})^{3}}\right)
$$

and the corresponding wave solution is

$$
E(x, y, z, t)=\frac{1}{2 \pi^{2} r}\left(\frac{1}{(v t-r-\mathrm{i})^{3}}-\frac{1}{(v t+r-\mathrm{i})^{3}}\right) .
$$

The obtained above analytical solutions (25), (27), (29), (31) and (33) of the linear wave equation (3) are spherically symmetric. Substituting the time variable $t=0$ in these solutions the initial conditions of the wave equation become exactly the corresponding localized smooth functions $V^{*}$. In these cases the initial functions decrease with the generation of outside and inside fronts and the energy densities distribute over the whole space radially. 


\section{Nonlinear Regime of Broad-Band Optical Pulses}

\subsection{Nonlinear Propagation of Broad-Band Pulses in Air. Lorentz Type Soliton}

As it was mentioned in [10], after neglecting two small perturbation terms, the corresponding nonlinear amplitude equation of broad-band femtosecond pulses can be reduced to

$$
\Delta C-\frac{1}{v^{2}} \frac{\partial^{2} C}{\partial t^{2}}+\gamma C^{3}=0
$$

where $\gamma=C_{0}^{2} k_{0}^{2} n_{2}$ is the nonlinear coefficient. equation (34) admits exact soliton solution propagating in forward direction only. The soliton solution of equation (34) is [10]

$$
C=\frac{\operatorname{sech}(\ln (\tilde{r}))}{\tilde{r}}=\frac{2}{1+\tilde{r}^{2}}
$$

where $\gamma=2$ and $\tilde{r}=\sqrt{x^{2}+y^{2}+(z+\mathrm{i} a)^{2}-v^{2}(t+\mathrm{i} a / v)^{2}}$. The main difference of the unstable solutions of the linear wave equation (3) and the amplitude equation (6) is that the soliton solution of the nonlinear wave equation (34) preserves its spatial and spectral shape in time. Thus, for the first time a photon-like propagation is obtained as exact solution of the corresponding $3 D+1$ nonlinear wave equation (34). The stable soliton solution of the one-dimensional nonlinear Schrödinger equation admits sech form, while the $3 D+1$ soliton solution of the nonlinear wave equation (34) has Lorentz shape with asymmetric $k_{z}$ spectrum. All above results are obtained for the cases of optical media with local linear and nonlinear response. Recently in [15] was demonstrated the existence of integrable regimes in nonlocal nonlinear optics using paraxial approximation. One including of the non-locality of the optical and magnetic response to a non-paraxial model will be performed in a next paper.

\subsection{Propagation of Broad-Band Pulses in Nonlinear Vacuum. Nonlinear Spher- ical Wave}

Our intention to find nonlinear solutions with angular momentum is a logical next step. In 1935 Euler and Kockel [4] predict one intrinsic nonlinearity of the electromagnetic vacuum due to the electron-positron nonlinear polarization. This leads to field-dependent dielectric tensor in the form

$$
\epsilon_{i k}=\delta_{i k}+\frac{7 e^{4} \hbar}{45 \pi m^{4} c^{7}}\left(2\left(|\vec{E}|^{2}-|\vec{B}|^{2}\right) \delta_{i k}+7 B_{i} B_{k}\right)
$$


where a complex form of the electrical $E_{i}$ and magnetic $B_{i}$ components is used. Note that the term containing $B_{i} B_{k}$ vanishes, when we investigate localized electromagnetic wave with only one magnetic component $B_{l}$. The dielectric response relevant to such optical pulse is thus

$$
\epsilon_{i k}=\delta_{i k}+\frac{14 e^{4} \hbar}{45 \pi m^{4} c^{7}}\left(|\vec{E}|^{2}-|\vec{B}|^{2}\right) \delta_{i k} .
$$

The magnetic field, rather than the electrical field, appears in the expression of the dielectric response (37) and the nonlinear addition to the energy distribution (effective mass density) of an electromagnetic wave in nonlinear vacuum can be expressed in electromagnetic units as $I_{n l}=\left(|\vec{E}|^{2}-|\vec{B}|^{2}\right)$. In the case when the spectral width of a pulse $\Delta k_{z}$ exceeds the values of the main wave-vector, i.e., $\Delta k_{z} \simeq k_{0}$, the system of amplitude equations in nonlinear vacuum becomes

$$
\begin{aligned}
& \Delta \vec{E}-\frac{1}{c^{2}} \frac{\partial^{2} \vec{E}}{\partial t^{2}}+\gamma\left(|\vec{E}|^{2}-|\vec{B}|^{2}\right) \vec{E}=0 \\
& \Delta \vec{B}-\frac{1}{c^{2}} \frac{\partial^{2} \vec{B}}{\partial t^{2}}+\gamma\left(|\vec{E}|^{2}-|\vec{B}|^{2}\right) \vec{B}=0
\end{aligned}
$$

where $\gamma=\frac{7 k_{0}^{2} e^{4} \hbar}{90 \pi m^{4} c^{7}}$ and $\vec{E}, \vec{B}$ are the amplitude functions. Initially, we can write the components of the electrical and the magnetic fields as a vector sum of circular and linear components

$$
E_{z}, \quad E_{c}=\mathrm{i} E_{x}-E_{y}, \quad B_{l}=-B_{z} .
$$

Thus (38) is transformed in the following system of scalar equations

$$
\begin{gathered}
\Delta E_{z}-\frac{1}{c^{2}} \frac{\partial^{2} E_{z}}{\partial t^{2}}+\gamma\left(\left|E_{z}\right|^{2}+\left|E_{c}\right|^{2}-\left|B_{l}\right|^{2}\right) E_{z}=0 \\
\Delta E_{c}-\frac{1}{c^{2}} \frac{\partial^{2} E_{c}}{\partial t^{2}}+\gamma\left(\left|E_{z}\right|^{2}+\left|E_{c}\right|^{2}-\left|B_{l}\right|^{2}\right) E_{c}=0 \\
\Delta B_{l}-\frac{1}{c^{2}} \frac{\partial^{2} B_{l}}{\partial t^{2}}+\gamma\left(\left|E_{z}\right|^{2}+\left|E_{c}\right|^{2}-\left|B_{l}\right|^{2}\right) B_{l}=0 .
\end{gathered}
$$

Let us now parameterize the $3 D+1$ space-time through pseudospherical coordinates $(r, \tau, \theta, \varphi)$

$$
\begin{array}{ll}
c t=r \sinh (\tau) & x=r \cosh (\tau) \sin (\theta) \cos (\varphi) \\
y=r \cosh (\tau) \sin (\theta) \sin (\varphi) & z=r \cosh (\tau) \cos (\theta)
\end{array}
$$

where $r=\sqrt{x^{2}+y^{2}+z^{2}-c^{2} t^{2}}$. 
After calculations the corresponding d'Alambert operator in pseudospherical coordinates becomes [14]

$$
\Delta-\frac{1}{c^{2}} \frac{\partial^{2}}{\partial t^{2}}=\frac{3}{r} \frac{\partial}{\partial r}+\frac{\partial^{2}}{\partial r^{2}}-\frac{1}{r^{2}} \frac{\partial^{2}}{\partial \tau^{2}}-2 \frac{\tanh \tau}{r^{2}} \frac{\partial}{\partial \tau}+\frac{1}{r^{2} \cosh ^{2} \tau} \Delta_{\theta, \varphi}
$$

where with $\Delta_{\theta, \varphi}$ is denoted the angular part of the usual Laplace operator. The system of equations (40) in pseudo-spherical then coordinates becomes

$$
\begin{aligned}
\frac{3}{r} \frac{\partial E_{z}}{\partial r} & +\frac{\partial^{2} E_{z}}{\partial r^{2}}-\frac{1}{r^{2}} \frac{\partial^{2} E_{z}}{\partial \tau^{2}}-2 \frac{\tanh \tau}{r^{2}} \frac{\partial E_{z}}{\partial \tau} \\
& +\frac{1}{r^{2} \cosh ^{2} \tau} \Delta_{\theta, \varphi} E_{z}+\gamma\left(\left|E_{z}\right|^{2}+\left|E_{c}\right|^{2}-\left|B_{l}\right|^{2}\right) E_{z}=0 \\
\frac{3}{r} \frac{\partial E_{c}}{\partial r} & +\frac{\partial^{2} E_{c}}{\partial r^{2}}-\frac{1}{r^{2}} \frac{\partial^{2} E_{c}}{\partial \tau^{2}}-2 \frac{\tanh \tau}{r^{2}} \frac{\partial E_{c}}{\partial \tau} \\
& +\frac{1}{r^{2} \cosh ^{2} \tau} \Delta_{\theta, \varphi} E_{c}+\gamma\left(\left|E_{z}\right|^{2}+\left|E_{c}\right|^{2}-\left|B_{l}\right|^{2}\right) E_{c}=0 \\
\frac{3}{r} \frac{\partial B_{l}}{\partial r} & +\frac{\partial^{2} B_{l}}{\partial r^{2}}-\frac{1}{r^{2}} \frac{\partial^{2} B_{l}}{\partial \tau^{2}}-2 \frac{\tanh \tau}{r^{2}} \frac{\partial B_{l}}{\partial \tau} \\
& +\frac{1}{r^{2} \cosh ^{2} \tau} \Delta_{\theta, \varphi} B_{l}+\gamma\left(\left|E_{z}\right|^{2}+\left|E_{c}\right|^{2}-\left|B_{l}\right|^{2}\right) B_{l}=0
\end{aligned}
$$

The equations (43) are solved using the method of separation of the variables

$$
E_{i}(r, \tau, \theta, \varphi)=R(r) T_{i}(\tau) Y_{i}(\theta, \varphi), \quad B_{l}(r, \tau, \theta, \varphi)=R(r) T_{l}(\tau) Y_{l}(\theta, \varphi)
$$

where $i=z, c$. We use an additional constrain on the angular and "spherical" time parts

$$
\left|T_{z}\right|^{2}\left|Y_{z}(\theta, \varphi)\right|^{2}+\left|T_{c}\right|^{2}\left|Y_{c}(\theta, \varphi)\right|^{2}-\left|T_{l}\right|^{2}\left|Y_{l}(\theta, \varphi)\right|^{2}=\text { const. }
$$

The condition (45) separates the variables. The nonlinear terms appear in the radial part only. Thus the radial parts obey the equation

$$
\frac{3}{r} \frac{\partial R}{\partial r}+\frac{\partial^{2} R}{\partial r^{2}}-\frac{A_{i}}{r^{2}} R+\gamma|R|^{2} R=0
$$

where $A_{i}$, for $i=z, c, l$ are separation constants. We look for solutions which possess more clearly expressed localization than the scalar soliton solution

$$
R=\frac{\operatorname{sech}\left(\ln \left(r^{\alpha}\right)\right)}{r}
$$


and where $\alpha, \gamma$ and the separation constants $A_{i}, i=z, c, l$ satisfy the relations

$$
\alpha^{2}-1=A_{i}, \quad 2 \alpha^{2}=\gamma
$$

The corresponding $\tau$ - dependent part of the equations (43) are linear

$$
\cosh ^{2} \tau \frac{\mathrm{d}^{2} T_{i}}{\mathrm{~d} \tau^{2}}+2 \sinh \tau \cosh \tau \frac{\mathrm{d} T_{i}}{\mathrm{~d} \tau}+\left(C_{i}-A_{i} \cosh ^{2} \tau\right) T_{i}=0
$$

where $i=z, c, l$ and $C_{i}$ are another separation constants connected with the angular part of the Laplace operator $Y_{i}(\theta, \varphi)$. Only the following solutions of the equation (49) which satisfy the condition (45) exist

$$
T_{z}=\cosh \tau, \quad T_{c}=\cosh \tau, \quad T_{l}=\sinh \tau
$$

with separation constants: for the electrical part $A_{z}=A_{c}=3, C_{z}=C_{c}=2$ and for the magnetic part $A_{l}=3, C_{l}=0$. Thus the magnetic part of the system of equations (43) do not depend on the angular components, i.e., $Y_{l}(\theta, \varphi)=0$, as for the electrical part $Y_{z}(\theta, \varphi), Y_{c}(\theta, \varphi)$ we have the following linear system of equations

$$
\frac{\Delta_{\theta, \varphi} Y_{i}}{Y_{i}}=-2
$$

where now $i=z, c$. There are only two solutions of the equation (51) which satisfy the condition (45)

$$
Y_{z}=\cos \theta, \quad Y_{c}=\sin \theta \exp (\mathrm{i} \varphi)
$$

Using the relation between the separation constants $A_{i}$ and the real number $\alpha$ we obtain

$$
\alpha^{2}=4, \quad \alpha= \pm 2, \quad \gamma=8
$$

Finally, we can write the explicit solution of the system of nonlinear equations (40) which describes the propagation of a electromagnetic wave in nonlinear vacuum

$$
\begin{aligned}
E_{z}(r, \tau, \theta) & =\frac{\operatorname{sech}\left(\ln \left(r^{ \pm 2}\right)\right)}{r} \cosh \tau \cos \theta \\
E_{c}(r, \tau, \theta, \varphi) & =\frac{\operatorname{sech}\left(\ln \left(r^{ \pm 2}\right)\right)}{r} \cosh \tau \sin \theta \exp (\mathrm{i} \varphi) \\
B_{l}(r, \tau) & =\frac{\operatorname{sech}\left(\ln \left(r^{ \pm 2}\right)\right)}{r} \sinh \tau
\end{aligned}
$$


If we rewrite the solution in the Cartesian coordinates, it is not difficult to show that the solution (54) of the system (40) admits finite energy and possesses its own angular momentum $l=1$

$$
\begin{aligned}
E_{z}(x, y, z, t) & =\frac{2 z}{r^{4}+1} \\
E_{c}(x, y, z, t) & =\frac{2(x+\mathrm{i} y)}{r^{4}+1} \\
B_{l}(x, y, z, t) & =\frac{2 c t}{r^{4}+1}
\end{aligned}
$$

where $r=\sqrt{x^{2}+y^{2}+z^{2}-c^{2} t^{2}}$ and $\hat{r}=\sqrt{x^{2}+y^{2}+z^{2}}$. The intensity profile of the solution now becomes

$$
I(x, y, z, t)=|\vec{E}|^{2}+|\vec{B}|^{2}=\frac{4\left(x^{2}+y^{2}+z^{2}+c^{2} t^{2}\right)}{\left(\left(x^{2}+y^{2}+z^{2}-c^{2} t^{2}\right)^{2}+1\right)^{2}} .
$$

This solution describes a nonlinear spherical shock wave.

\section{Conclusions}

In this paper the wave equation (3) is solved by using a simple form of the CourantHilbert ansatz (2). In this way we reduce the equation (3) to a parabolic type diffraction equation (6) for the envelope. The corresponding initial value problem can be solved and different kind of finite energy exact solutions can be found of the differential equation (6) as well as of the wave equation (3). The solutions spread with the velocity of light and possess different evolution. In the laser optics a Gaussian light bullet, or another kind of initial amplitude functions $V(x, y, z, 0)$ are localized near one monochromatic wave $E(x, y, z, 0)=\exp \left(\mathrm{i} k_{0} z\right) V(x, y, z, 0)$ and they are in fact a superposition of plane waves near one main wave-number. In the case when such pulses have a lot of cycles under the envelope they broaden mainly in transverse direction following the Fresnel's law of diffraction. The evolution is exactly identical with the laser pulse dynamics measured in the experiments. The latest experimental [16] and theoretical [11] observations of a few, single and half-cycle pulses reveal that at small diffraction lengths their intensity profile takes parabolic or semi-spherical form, i.e., the paraxial optics does not work for few, single and HCP's waves. If the initial pulses are not localized near a plane wave and are spherically symmetric the solutions of the wave equation (3) spread radially forming inside and outside wave fronts. These conclusions are based on the exact analytical solutions of both the wave equation and the amplitude equation 
presented in the paper. The initially localized amplitude functions decrease and the energy density of all obtained solutions distributes over whole space for a finite time. In nonlinear regime we have solved nonlinear wave equation and a system of nonlinear wave equations (40) governing propagation of broad-band optical pulses in nonlinear vacuum. The main difference between the solutions of the linear wave equation (3) and the amplitude equation (6), which spread in space, is that the soliton solution of the nonlinear wave equation (34) preserves its spatial and spectral shape in time. Thus, one photon-like propagation is obtained as an exact solution of the corresponding $3 D+1$ nonlinear wave equation (34) [10]. The scalar $3 D+1$ soliton solution of (34) has Lorentz shape with asymmetric $k_{z}$ spectrum. The solution (54) of the nonlinear vacuum system of equations (40) has a strong localization than scalar case and admits its own angular momentum.

\section{Acknowledgements}

This work is partially supported by the Bulgarian Science Foundation under grant DDVU 02/71.

\section{References}

[1] Abramochkin E. and Volostnikov V., Spiral Light Beams, Phys. Usp. 47 (2004) 1177-1203.

[2] Bandres M., Vector Helmholtz-Gauss and Vector Laplace-Gauss Beams, Opt. Lett. 30 (2005) 2155-2158.

[3] Courant R. and Hilbert D., Methods of Mathematical Physics, Interscience, New York 1962.

[4] Euler H. and Kockel K., Über die Streuung von Licht an Licht nach der Diracschen Theorie, Naturewiss 23 (1935) 246-253.

[5] Fock V., Electromagnetic Diffraction and Propagation Problems, Nauka, Moscow 1970.

[6] Gori F., Guattari C. and Padovani C., Bessel-Qauss Bams, Opt. Commun. 64 (1986) 491-495.

[7] Gutiérrez-Vega J. and Bandres M., Helmholtz-Gauss Waves, J. Opt. Soc. Am. A 22 (2005) 289-298.

[8] Kaplan A., Straub S. and Shkolnikov P., Electromagnetic Bubble Generation by Half-Cycle Pulses, Opt. Lett. 22 (1997) 405-407.

[9] Kiselev A., New Structures in Paraxial Gaussian Beams, Opt. Spektrosk. 96 (2004) 533-535. 
[10] Kovachev L., New Mechanism for THz Oscillation of the Nonlinear Refractive Index in Air: Particle-Like Solutions, J. Mod. Opt. 56 (2009) 1797 - 1803.

[11] Kovachev L. and Kovachev K., Diffraction of Femtosecond Pulses: Nonparaxial regime, JOSA A 25 (2008) 2232-2243; Erratum, JOSA A 25 (2008) 3097-3098.

[12] Leontovich M., On a Method of Solving Problems of the Propagation of Electromagnetic Waves, Izv. Akad. Nauk SSSR Ser. Fiz. 8 (1944) 16-22.

[13] Leontovich M. and Fock V., Solution of the Problem of Electromagnetic Waves Along the Earth's Surface by the Method of Parabolic Equation, J. Phys. USSR 10 (1946) 13-24.

[14] Lokas E., Positive Frequency Solutions of the Klein-Gordon Equation in the $N$-dimmenisonal de Sitter Spacetime, Acta Phys. Polonica B 26 (1995) 19-33.

[15] Moro L. and Konopelchenko B., High Frequency Integrable Regimes in Nonlocal Nonlinear Optics, J. Geom. Symmetry Phys. 7 (2006) 37-83.

[16] Naumova N., Nees J., Power E. and Mourou G., Attosecond Phenomena in the Relativistic $\lambda^{3}$ Regime, Laser Physics 15 (2005) 832-837.

[17] Soskin M. and Vasnetsov M., Singular Optics, Progress in Optics 42 (2001) 219-276.

[18] Zeibel J., Manipulating Wavepackets Dynamics with Half-Cycle Pulses, PhD Thesis, University of Virginia 2003.

Lubomir M. Kovachev

Institute of Electronics

Bulgarian Academy of Sciences

72, Tzarigradcko shossee

Sofia 1784, BULGARIA

E-mail address: lubomirkovach@yahoo.com

Daniela A. Georgieva

Faculty of Applied Mathematics and Computer Science

Technical University of Sofia

8, Kliment Ohridski Blvd.

Sofia 1000, BULGARIA

E-mail address: dgeorgieva@tu-sofia.bg 